\title{
Evaluation of the Pyrrolizidine Alkaloid Induced Liver Disease (PAILD) Active Surveillance System in Tigray, Ethiopia
}

\author{
Cindy Chiu*1, Danielle Buttke ${ }^{1}$, Girmay Welde², Richard Luce ${ }^{3}$, Asfaw Debela ${ }^{4}$, Amsalu \\ Bitew $^{5}$, Tesfaye Bayleyegn ${ }^{1}$, Sara Vagi ${ }^{1}$, Matthew Murphy ${ }^{1}$, Daniel Woldemichael ${ }^{2}$, Teshale \\ Seboxa ${ }^{6}$, Gidey G. Libanos ${ }^{7}$, Zeyeda Beyene ${ }^{7}$, Yohannes G. Hawaria ${ }^{7}$, Daddi Jimma ${ }^{4}$, Israel \\ Tareke $^{8}$, Danielle Rentz ${ }^{1}$ and Colleen Martin ${ }^{1}$
}

${ }^{1}$ Centers for Disease Control and Prevention, Atlanta, GA, USA; ${ }^{2}$ Tigray Agriculture Bureau, Tigray, Ethiopia; ${ }^{3}$ Centers for Disease Control and Prevention, Addis Ababa, Ethiopia; ${ }^{4}$ Ethiopian Health and Nutrition Research Institute, Addis Ababa, Ethiopia; ${ }^{5}$ Suhul Hospital, Tigray, Ethiopia; ${ }^{6}$ Addis Ababa University, Addis Ababa, Ethiopia; ${ }^{7}$ Tigray Regional Health Bureau, Tigray, Ethiopia; ${ }^{8}$ World Health Organization, Addis Ababa, Ethiopia

\section{Objective}

To describe the results of the evaluation of the PAILD active surveillance system and lessons learned for similar surveillance efforts in a resource-limited setting.

\section{Introduction}

A liver disease of unknown etiology, called unknown liver disease (ULD) by the community, was first identified in 2002 in Tigray; a rugged, semi-arid, mountainous region that is considered one of the most drought-prone and food insecure regions of Ethiopia. ULD is a chronic condition characterized by epigastric pain, abdominal distention, ascites, emaciation, and hepato/splenomegaly. In 2005, the Ethiopian Health and Nutritional Research Institute was assigned by the Ethiopia Ministry of Health to assist the Tigray Regional Health Bureau and oversee the disease investigation. In 2008, Centers for Disease Control and Prevention (CDC) assisted the Ethiopian team and jointly developed the surveillance tools. The surveillance system was implemented in 2009 with the objectives to determine the magnitude and distribution of the disease; identify disease trends; detect cases to provide them with clinical care; and inform health officials and funding bodies for resource allocation. After several investigations, a local plant containing a particular type of pyrrolizidine alkaloid (PA) toxin that contaminated local foodstuffs was identified as the etiologic agent, and ULD was renamed PAILD in 2011.

\section{Methods}

From 20 September to 1 October 2011, we conducted site visits, held semi-structured interviews with 20 staff members, reviewed reporting materials, and summarized the information flow including data collection, reporting, analysis, and dissemination.

\section{Results}

This surveillance system was implemented in 13 rural, resourcelimited districts in the NW, Central and Western Zones. The system identified a total of 1033 cases, including 314 deaths, as of September 2011; guided medication distribution to the health facilities; served as a registry for patient follow up; and provided decision-mak- ers with information needed to allocate resources. A large-scale training was conducted in 2010; however, high staff turnover and a lack of backup surveillance staff at each site suggested that additional training may be needed. Due to the absence of a diagnostic test, the case definition was very simple to enable frontline staff in the communities and at the health posts/centers to identify disease cases. These individuals travelled long distances by foot to deliver paper surveillance forms to the district health offices. A surveillance team placed in the NW Zonal office collected missing reports from the health facilities given limited transportation; however they have left since this evaluation. Information from the surveillance system was shared with partner agencies at the national level every 3 to 6 months; however, this information was not shared with frontline staff.

\section{Conclusions}

The PAILD Active Surveillance System met its objectives as originally defined. Evaluation of this unique surveillance system for a chronic disease with unknown cause in a resource-limited setting provides several lessons that can inform similar surveillance efforts. Ongoing logistical challenges (e.g., shortage of paper forms, lack of transportation, and long distances between locations) complicated data collection and reporting. While electronic reporting may have helped overcome some of these difficulties, it was not feasible in this setting. Frontline staff identified cases in the community so that they could receive treatment; these key staff can be further incentivized by receiving regular training and surveillance reports. Ongoing support will be critical to overcome these unique challenges to ensure continual disease monitoring as interventions to disrupt PA exposure are implemented in the community.

\section{Keywords}

Evaluation; Pyrrolizidine Alkaloid Induced Liver Disease; Active Surveillance System; Ethiopia
*Cindy Chiu
E-mail: vic2@cdc.gov 